\title{
Validación de requisitos no funcionales bajo el enfoque de la gestión del conocimiento
}

\section{Validation of non-functional requirements under the knowledge management approach}

\author{
Sandra Buitrón Ruiz \\ Luis Medina Erazo \\ Juan Bolaños Benítez \\ Francisco Pino Correa \\ Universidad del Cauca, Colombia
}

\section{OPEN ACCESS}

Recibido: 22/09/2020

Aceptado: 22/10/2020

Publicado: 23/11/2020

Correspondencia de autores: sandrabr@unicauca.edu.co
(1)

b

pror

.

de cons Conclusiones: El proceso de validación integra elementos concretos de la gestión de conocimiento, y permite la simplificación de la identificación, captura, especificación e incluso el análisis, de los requisitos no funcionales, al poder establecer un formato único y estructurado el cual puede ser gestionado durante la ejecución de éstas actividades.

Palabras clave: Requisitos no funcionales; gestión del conocimiento; validación de requisitos, focus group

Abstract

Objective: Requirements validation is an important software development activity since it allows to business and technical stakeholders agree on functional and non-functional software requirements in order to these being complete, consistent and unambiguous. An appropriate non-functional requirements validation supports to software product quality. Methodology: A conceptual model and a process for non-functional requirements validation are proposed using the action-research methodology multicycle with bifurcation. Results: Through the focus group method that involved professionals with experience in requirements validation this proposal has been preliminarily evaluated and positive results regarding the relevance, applicability and clarity of the validation process has been obtained. Conclusions: The validation process integrates specific elements of knowledge management allowing the simplification of the identification, capture, specification and even non-functional requirements analysis, by establishing a structured unique format which can be managed during the execution of these practices.

Keywords: Non-functional requirements, knowledge management, requirement validation, focus group.

Como citar (IEEE): S. Buitrón-Ruiz., L. Medina-Erazo., J. Bolañoz-Benítez., y F. Pino-Correa. “Validación de requisitos no funcionales bajo el enfoque de la gestión del conocimiento" vol. 8, n³, pp. 50-71, 2020. DOI: https://doi.org/10.17081/invinno.8.3.4704 


\section{Introducción}

La ingeniería de software tiene como objetivo lograr productos de calidad, la cual se determina a través de la calidad de diseño y fabricación del producto [1,2]. Esta calidad viene determinada a través del cumplimiento de los requisitos del producto software, los cuales son la descripción de los servicios proporcionados por el sistema y sus restricciones operativas [3]. Estas restricciones deben se recopiladas y analizadas para una posterior validación acertada de los requisitos [4], de manera que se pueda asegurar que los éstos han sido declarados de manera excelente (completos, no ambiguos, correctos, factibles, necesarios, priorizados, inequívocos y verificables) y que las especificaciones también son completas, consistentes, modificables y trazables [5,6]. Sin embargo, y pese a la relevancia del propósito de esta actividad de validación de requisitos en la calidad del producto software, es investigada de manera pequeña y con tendencia decreciente [7].

Dentro de esta validación es necesario integrar los Requisitos No Funcionales (RNF) de manera que también puedan ser expresados o representados de manera adecuada [8]; el uso del lenguaje natural y formatos libres para representar los requisitos pueden generar imprecisiones y a menudo propenden a la incorporación de errores [9]. Entre las dificultades generadas por un proceso inadecuado de validación de requisitos, incluyendo los RNF, se encuentran: (i) falta de conformidad con las normas de calidad, requisitos ambiguos, errores en el modelo del sistema y conflicto en los requisitos que no fueron detectados durante el proceso de análisis [10], (ii) errores humanos simples en la captura, mal diseño del documento de requisitos y requisitos mal definidos [11], y (iii) mayor generación de solicitudes de cambio a partir de fallas en las validaciones previas [12]. La inadecuada especificación de los RNF conlleva una mayor necesidad de ser validados durante su proceso de desarrollo, de manera que se compruebe su no ambigüedad, coherencia, integridad y exactitud [10]

Las dificultades descritas anteriormente y las implicaciones que tienen los RNF sobre el comportamiento y el desarrollo del producto de software [11], son el problema que se pretende abordar en el presente artículo, buscando solucionarlo mediante un proceso de validación para RNF estructurado desde la perspectiva de la gestión de conocimiento. La gestión de conocimiento, ha sido reconocida en los últimos años como disciplina esencial para la industria del software $[13,14]$, por lo cual ha sido considerada como fundamento de la presente investigación. En este artículo se propone: (i) un modelo conceptual del proceso de validación para requisitos no funcionales, para lo cual se identificaron elementos relevantes desde la literatura, los cuales fueron analizados en términos de: criterios, técnicas, métricas y actividades para la validación de estos requisitos, y (ii) un proceso de validación de requisitos no funcionales en el que participan activamente los interesados mediante la incorporación de elementos de gestión del conocimiento, tales como flujos de conocimiento, ciclos de gestión de conocimiento y tipos de conocimiento.

El proceso para la validación de RNF se centra en gestionar el conocimiento acerca de estos requisitos, a través de actividades que permiten la transformación, creación y disposición de este conocimiento $[15,16]$. El proceso propuesto busca aportar al que hacer de los proyectos de desarrollo de software, ofreciendo una manera de integrar los RNF en las dinámicas de validación. Este proceso ha sido evaluado preliminarmente, a través del método de Focus Group en el cual participaron profesionales con experiencia en el área de validación de requisitos, obteniendo resultados positivos frente a la relevancia, aplicabilidad y claridad del mismo en el contexto de organizaciones desarrolladoras de software.

Además de esta introducción, el articulo presenta en la sección 2, la metodología utilizada para la investigación, en la sección 3, los trabajos relacionados con la validación de requisitos, en la sección 4, la propuesta de validación de requisitos no funcionales, en la sección 5 , el diseño y resultados de la aplicación del focus group con el que se evalúo la propuesta, y en la sección 6 , las conclusiones y trabajos futuros. 


\section{Metodología}

\section{Estrategia de investigación}

Para llevar a cabo el trabajo de investigación se utilizó la metodología investigación acción multi-ciclo con bifurcación [17], dentro de la cual se realizaron los ciclos: conceptual, metodológico y de evaluación. Cada ciclo permite abordar actividades investigativas con el fin desarrollar la propuesta de manera sistemática.

En el ciclo conceptual, se analiza el estado del arte del proceso de validación de RNF y su impacto sobre el proceso de desarrollo de software. Adicionalmente, se revisan las características de calidad que se tienen en cuenta para el proceso de validación de RNF y se analiza la literatura existente con respecto a los mecanismos y técnicas utilizadas para realizar este proceso de validación. Durante el ciclo metodológico, se define la propuesta, a partir de la información recolectada en el ciclo previo de la metodología, en términos de dos elementos: (i) un proceso de validación de requisitos no funcionales, y (ii) elementos de gestión del conocimiento que aportan en la validación de este tipo de requisitos. Estos elementos se relacionan de manera armonizada dentro del proceso de validación de RNF. En el ciclo de evaluación, se diseña un Focus Group con expertos en el proceso de validación de requisitos, quienes a través de su experiencia en proyectos de desarrollo de software brinden una retroalimentación acerca de la idoneidad de la propuesta, tanto desde la perspectiva técnica como desde la perspectiva de negocio.

\section{Trabajos relacionados}

Para identificar las propuestas frente al tema de investigación, se realiza una revisión de la literatura siguiendo algunas de las actividades propuestas en [18]. Para esta revisión, se define: (i) una ventana de tiempo de 18 años, (ii) utilizar las bases de datos Scopus (la cual incluye bases de datos como ACM, IEEEExplore, Springer y ScienceDirect) y Google Scholar, y (iii) ejecutar las siguientes cadenas de búsqueda: "requirements validation" AND "non-functional requirements" AND "knowledge Management", "software engineering" AND "requirements validation", "requirements validation" AND "non-functional requirements", y "requirements validation" AND "functional requirements".

A partir de las propuestas encontradas en revistas, memorias de congresos, memorias de simposios y reportes técnicos, se obtuvo 38 artículos relevantes, los cuales se determinaron a través del análisis y de la aplicación de los siguientes criterios de inclusión: (i) las propuestas debían relacionar las características de calidad del software, y (ii) debían estar enfocados en contribuir al entendimiento de los requisitos (funciones o no funcionales) durante su validación. Finalmente, a partir de los artículos relevantes, se excluyó los que no tuvieran aporte directo sobre el proceso de validación de requisitos; obteniendo finalmente 12 artículos primarios. A continuación, se describen, en términos generales, estas propuestas:

En [19], se presenta un enfoque que busca validar los requisitos escritos en lenguaje natural, a través de la comprobación de las abstracciones del documento de requisitos, [20] propone un framework para la ingeniería de requisitos basada en el conocimiento (KBRE), en el cual el conocimiento del dominio y la semántica de los requisitos son fundamentales para la elaboración, estructuración y gestión de los requisitos capturados, incluyendo las inconsistencias en los requisitos. En [8], se describen los resultados de experimentos sobre el uso de pruebas de aceptación como apoyo para aclarar los requisitos, centrándose en las tablas Fit (Framework for Integrated Test) como una manera de estructurar las pruebas de aceptación, las cuales se pueden traducir automáticamente en los casos de prueba ejecutables. [21] propone un marco teórico metodológico de técnicas para verificar y validar especificaciones de requisitos de software, que ayudará a definir los límites del sistema al momento de formular los requisitos, controlar 
y optimizar los procesos, y proveer al grupo de desarrollo una base para la estimación del tiempo y costo del desarrollo. De otro lado, [22] detalla un enfoque para el modelado y verificación de los requisitos en sistemas autoadaptables, [23] una técnica para la validación de modelos de comportamiento, [24] técnicas de validación de la seguridad y la fiabilidad del software, [25] técnicas teóricas de validación, [26] un enfoque para validar los requisitos de forma automática y apoyar el diseño multidimensional de bodega de datos, [27] una metodología para evaluar la calidad de los requisitos de forma automática, emulando la valoración de un experto en calidad. Finalmente, [28] propone que la determinación de los requisitos funcionales de software pueden ser validados mediante el modelado de estrategias de negocio (FBCM - Hechos basados en metodología de Modelado de Colaboración) usando las observaciones de campo y métodos de análisis de datos de tipo indicador.

Sin embargo, y pese a éstas investigaciones, se observa que no hay propuestas centradas en la validación de Requisitos No Funcionales, y adicionalmente, que el proceso de validación no ha sido abordado desde la perspectiva de gestión de conocimiento, de manera que, a través de elementos concretos de ésta disciplina se pueda llevar a cabo una validación más adecuada de éste tipo de requisitos, involucrando tanto a interesados técnicos como de negocio.

\section{Resultados}

\section{Validación de requisitos no funcionales}

A continuación, se presenta la propuesta de investigación, la cual se constituye de un modelo conceptual de validación de RNF y un proceso de validación de RNF.

\section{Modelo conceptual de la validación de requisitos no funcionales}

Posterior al proceso de análisis de la literatura, se estructura el modelo conceptual que soporta la propuesta para el proceso de validación de RNF (Figura 1), el cual se apoya en los siguientes dos pilares fundamentales: (i) las técnicas de validación de requisitos, las cuales soportan desde las diferentes maneras de realizar la validación, y (ii) la gestión del conocimiento, la cual, a través de conceptos como ciclos de conocimiento, flujos de conocimiento y tipos de conocimientos permite determinar las estrategias de validación más adecuadas para interactuar con cada uno de los stakeholders. De esta manera, este modelo conceptual busca guiar la correcta ejecución del proceso de validación de RNF en los proyectos

de desarrollo de software mediante la identificación y relación de criterios, técnicas y métricas para la validación de estos requisitos. 
Figura 1. Modelo conceptual al proceso para la validación de RNF

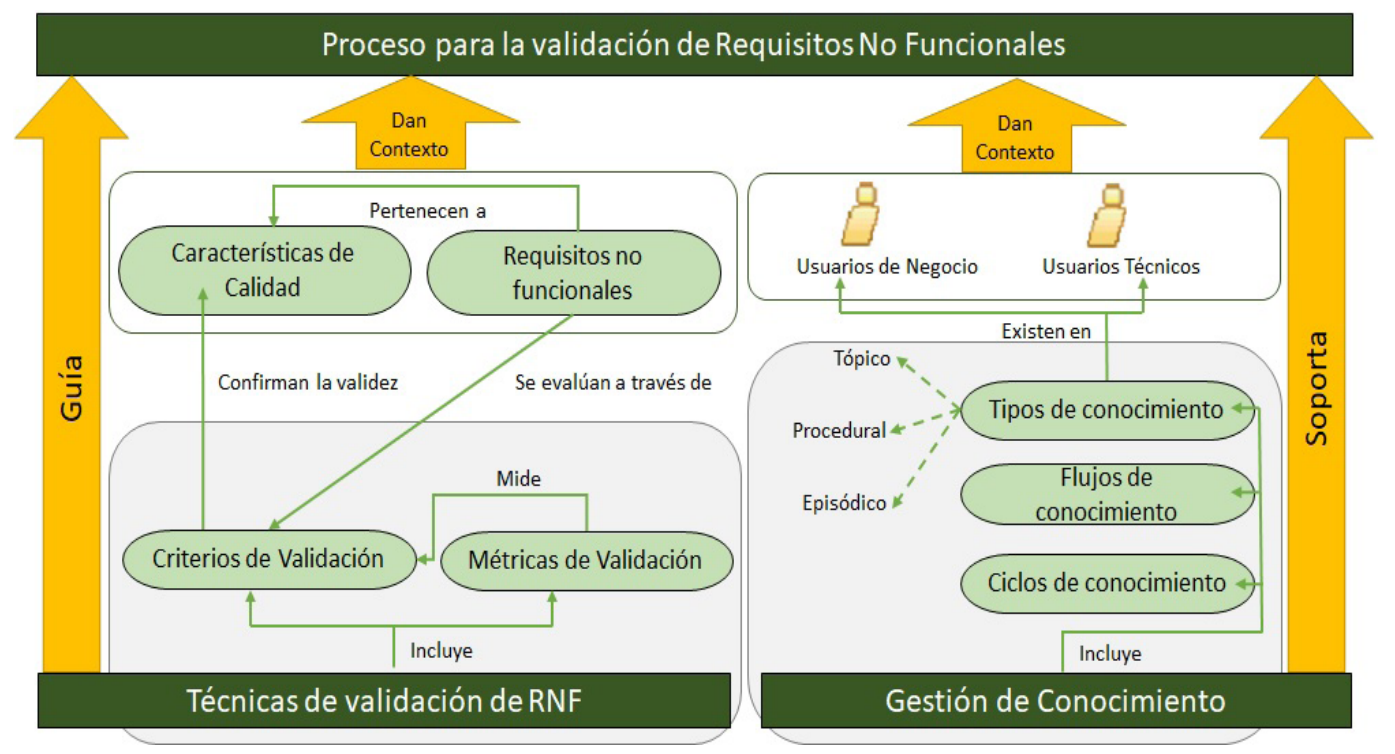

Fuente: Elaboración propia

\section{Criterios utilizados para la validación de requisitos no funcionales}

A partir de los artículos primarios encontrados en la revisión de la literatura, se inició por identificar y determinar los aspectos que se tenían en cuenta para realizar la validación de los requisitos, así como determinar los actores o participantes de éste proceso. En la Tabla 1, se muestra esta información extraída de las propuestas analizadas.

Tabla 1. Aspectos de validación sobre requisitos encontrados en la literatura

\begin{tabular}{|c|c|c|}
\hline Ref. & Qué se valida de los requisitos & Actores involucrados \\
\hline [19] & Exactitud, consistencia, minimalidad & $\begin{array}{l}\text { - Ingeniero de requisitos } \\
\text { - Cliente }\end{array}$ \\
\hline [22] & Inconsistentes & $\begin{array}{l}\text { - Ingeniero de requisitos } \\
\text { - Elicitador }\end{array}$ \\
\hline [23] & Consistente, completa, exactitud & - Observador \\
\hline [24] & Seguridad, fiabilidad & $\begin{array}{l}\cdot \text { Cliente } \\
\cdot \text { Ingeniero }\end{array}$ \\
\hline [21] & No ambigua, completa, correcta, verificable & $\begin{array}{l}\cdot \text { Cliente } \\
\cdot \text { Ingeniero }\end{array}$ \\
\hline [25] & Consistentes, completos & $\begin{array}{l}\text { - Desarrolladores } \\
\text { - Cliente }\end{array}$ \\
\hline [26] & Completa, correcta & $\begin{array}{l}\text { - Ingeniero de requisitos } \\
\text { - Elicitador }\end{array}$ \\
\hline Ref. & Qué se valida de los requisitos & Actores involucrados \\
\hline [28] & Correcta & $\begin{array}{l}\text { - Ingeniero } \\
\text { - Usuario }\end{array}$ \\
\hline
\end{tabular}




\begin{tabular}{|l|l|l|}
\hline$[8]$ & $\begin{array}{l}\text { Ambigüedad, completitud, ausentes, de gran tamaño, sobre } \\
\text { especificados, muy genéricos, basados en una ilusión. }\end{array}$ & $\begin{array}{l}\text {-Usuarios finales } \\
\cdot \text { Elicitadores }\end{array}$ \\
\hline$[27]$ & Consistencia, ambigüedad, trazabilidad, precisión, atomicidad & -Ingeniero de requisitos. \\
\hline$[29]$ & Consistencia, completos & $\cdot$ Profesionales de software. \\
\hline$[20]$ & Consistencia, redundancia, solapamiento (overlaps) & $\begin{array}{l}\text { - Ingeniero de requisitos } \\
\cdot \text { Elicitador }\end{array}$ \\
\hline
\end{tabular}

\section{Fuente: Elaboración propia}

A partir de estos aspectos, se realizó un proceso de análisis conceptual para consolidar las definiciones utilizadas por los diferentes autores, considerando que estas definiciones fueran excluyentes con respecto a las demás. Posteriormente, se seleccionaron las definiciones valoradas como más claras y completas, para ser tomadas como las definiciones base de los criterios de validación para requisitos no funcionales de la propuesta (Tabla 2).

\section{Tabla 2. Criterios usados en la validación de los RNF}

\begin{tabular}{|c|c|}
\hline Criterios de validación & Definición \\
\hline No ambigüedad & Cada requisito tiene una sola interpretación. \\
\hline Concisos & Su redacción debe ser simple y clara para aquellos que vayan a consultarlo en un futuro. \\
\hline Completitud & $\begin{array}{l}\text { El requisito establecido no necesita mayor amplificación, ya que es medible y describe } \\
\text { suficientemente la capacidad y características para satisfacer las necesidades de las partes } \\
\text { interesadas. }\end{array}$ \\
\hline Consistencia & Un requisito es consistente si no es contradictorio con otro requisito. \\
\hline Verificabilidad & $\begin{array}{l}\text { El requisito tiene los medios para probar que el sistema cumple con el requisito especificado. La } \\
\text { evidencia recogida demuestra que el sistema puede satisfacer el requisito especificado. }\end{array}$ \\
\hline Trazabilidad & $\begin{array}{l}\text { Un requisito es trazable si se conoce su origen y se facilita su referencia a los componentes del } \\
\text { diseño y de la implementación. La trazabilidad hacia atrás indica el origen (documento, persona, } \\
\text { etc.) de cada requisito. La trazabilidad hacia delante de un requisito indica qué componentes del } \\
\text { sistema son los que realizan el requisito. }\end{array}$ \\
\hline Factibilidad & $\begin{array}{l}\text { Al usar el conocimiento de la tecnología existente los requisitos deben comprobarse para garantizar } \\
\text { que en realidad pueden implementarse. Dichas comprobaciones también tienen que considerar el } \\
\text { presupuesto y la fecha para el desarrollo del sistema. }\end{array}$ \\
\hline
\end{tabular}

\section{Fuente: Elaboración propia}

Para esta consolidación, adicionalmente, se tuvo en cuenta las siguientes fuentes, entre las cuales hay estándares internacionales: [30,31,32,28,33]. Con respecto a los actores involucrados en un proceso de validación de requisitos se concluye que son dos los actores fundamentales: (i) un actor técnico que conoce y guía el proceso de validación de RNF y un actor de negocio que participa activamente en éste proceso; estos dos actores, serán llamados Facilitador (profesional en ingeniería) e Stakeholder (usuarios finales y usuarios de negocio) respectivamente en la propuesta. 


\section{Técnicas de validación aplicables para requisitos no funcionales}

Del análisis de la literatura, también se extrae información sobre las técnicas de validación de requisitos, identificando las técnicas que los autores proponen utilizar en el proceso de validación de requisitos. La información extraída se organiza de manera que se obtiene un conjunto de técnicas de validación por cada una o varias de las características de calidad (requisitos no funcionales) de la ISO 25010 [1]. Lo que se evidencia en la literatura es que predomina la aplicación de las técnicas de validación en determinada situación y dependiendo del contexto en el que se encuentre la organización y la importancia que se le dé a cada una de las características de calidad.

Estas técnicas de validación se presentan bajo una estructura que las relaciona con las características de calidad del estándar, para que, dependiendo del nivel de conocimiento de los stakeholders frente a lo que conocen de los RNF, el facilitador del proceso de validación seleccione la técnica más adecuada para la realización del mismo (Tabla 3).

Tabla 3. Características y sub-características de calidad ISO 25010 versus Técnicas de validación de RNF

\begin{tabular}{|c|c|c|}
\hline $\begin{array}{c}\text { Característica } \\
\text { de calidad }\end{array}$ & Sub-caracteristicas & Técnica de validación \\
\hline \multirow{3}{*}{$\begin{array}{l}\text { Eficiencia de } \\
\text { desempeño }\end{array}$} & - Comportamiento temporal. & - Validación Cruzada (Escenarios) \\
\hline & - Utilización de recursos. & - Revisión Técnica Formal (RTF) \\
\hline & - Capacidad. & - Auditorías \\
\hline \multirow{2}{*}{ Compatibilidad } & -Coexistencia. & - RTF \\
\hline & - Interoperabilidad. & - Auditorías \\
\hline \multirow{6}{*}{ Usabilidad } & - Capacidad para reconocer su adecuación. & - Validación Cruzada (Escenarios) \\
\hline & - Capacidad de aprendizaje. & -Prototipos \\
\hline & - Capacidad para ser usado. & - RTF \\
\hline & - Protección contra errores de usuario. & - Auditorías \\
\hline & - Estética de la interfaz de usuario. & -Glosario \\
\hline & - Accesibilidad. & \\
\hline $\begin{array}{c}\text { Característica } \\
\text { de calidad }\end{array}$ & Sub-caracteristicas & Técnica de validación \\
\hline \multirow{4}{*}{ Fiabilidad } & - Madurez. & - Técnicas tradicionales de análisis estático SFMEA y SFTA. \\
\hline & - Disponibilidad. & - Validación Cruzada (Escenarios) \\
\hline & - Tolerancia a fallos. & - RTF \\
\hline & - Capacidad de recuperación. & - Auditorías \\
\hline
\end{tabular}




\begin{tabular}{|c|c|c|}
\hline \multirow{5}{*}{ Seguridad } & - Confidencialidad. & - Técnicas tradicionales de análisis estático SFMEA y SFTA \\
\hline & - Integridad. & - Validación Cruzada (Escenarios) \\
\hline & - No repudio. & - RTF \\
\hline & - Responsabilidad. & - Auditorías \\
\hline & - Autenticidad. & -Glosario \\
\hline \multirow{5}{*}{ Mantenibilidad } & - Modularidad. & - Validación Cruzada (Escenarios) \\
\hline & - Reusabilidad. & - RTF \\
\hline & - Analizabilidad. & - Auditorías \\
\hline & - Capacidad para ser modificado. & -Glosario \\
\hline & - Capacidad para ser probado. & \\
\hline \multirow{3}{*}{ Portabilidad } & - Adaptabilidad. & - RTF \\
\hline & - Capacidad para ser instalado. & - Validación Cruzada (Escenarios) \\
\hline & - Capacidad para ser reemplazado. & - Auditorías \\
\hline
\end{tabular}

\section{Fuente: Elaboración propia}

Esta estructura tiene como propósito apoyar la selección de la técnica de validación de acuerdo al requisito no funcional que se esté trabajando a partir de la información registrada en los documentos de especificación del producto software. Las técnicas de validación de RNF son un soporte fundamental para esta propuesta, debido a que ayudan a los stakeholders a tener un mejor entendimiento sobre los RNF, y con ello poder realizar una mejor validación de los mismos, permitiendo realizar de manera concreta y satisfactoria su validación.

\section{Métricas para la validación de requisitos no funcionales}

A pesar de identificar diferentes técnicas de validación de requisitos, no se encontró en la literatura métricas relacionadas específicamente con la validación de RNF, por lo cual se decide proponer unas métricas genéricas dentro del proceso, como mecanismo de apoyo para determinar el cumplimiento de cada uno de los criterios de validación estructurados en la Tabla 2 frente al documento de especificación de RNF. Para establecer dichas métricas de validación se utilizó como referencia la norma ISO 25023 [34] y se tomó, para los fines especificados en cada criterio de validación, la fórmula $X=1-A / B$, donde $A$, es el número de requisitos no funcionales que no cumplan con el criterio de validación respectivo (por ejemplo el criterio de concisos), y B, el número total de RNF a validar para el producto. Este resultado permitirá responder a preguntas específicas como por ejemplo ¿Cuán concisos son los RNF especificados? En la Tabla 4 se detalla la información acerca del tipo de escala, tipo de medida y entradas que se requieren para las métricas de acuerdo al estándar mencionado. 
Tabla 4. Convenciones para las métricas propuestas

\begin{tabular}{|c|c|c|}
\hline Tipo de escala & Tipos de medida & Entradas a la medición \\
\hline $0<=X<=1$ & \multirow{2}{*}{ Absolutas } & $A=$ cantidad contada de RNF que no cumplen \\
\hline Cuanto más cerca de 1, mejor & & $\mathrm{B}=$ cantidad total de RNF a validar en el producto \\
\hline \multicolumn{3}{|c|}{$\begin{array}{l}\text { NOTA: La entrada al proceso de medición es la especificación de requisitos actualizada. Cualquier cambio identificado durante el ciclo } \\
\text { de vida debe aplicarse a las especificaciones de los requisitos antes de utilizarlo en el proceso de medición. }\end{array}$} \\
\hline
\end{tabular}

Fuente: Adaptado de [34]

Luego de calcular las métricas específicas a cada criterio de validación, se propone realizar un promedio entre todos éstos resultados para así obtener un solo valor de qué tan correcta se encuentra la especificación de $\mathrm{RNF}$, considerando que todos estos criterios tienen igual importancia para el contexto en el que se realice la validación. Finalmente, con el valor obtenido, el cual tendrá la misma escala y convenciones definidas en la Tabla 4, se analizará el rango en el que se encuentre la especificación de RNF y será aceptada o rechazada. A modo de ejemplo, en este trabajo de investigación se propone la siguiente escala de interpretación: $0.0<=x<=0.66$ Rechazada, $0.67<=x<=1.00$ Aceptada. La escala podrá ser modificada dependiendo de las necesidades de la organización para tomar una decisión frente a la especificación de los RNF.

\section{Proceso de validación de requisitos no funcionales}

A partir del modelo conceptual y buscando instrumentalizar su aplicación en proyectos reales de desarrollo de software, se define un proceso para la validación de RNF, el cual se describe en términos de objetivo del proceso, diagrama de actividades del proceso y (iii) elementos de gestión de conocimiento. El objetivo del proceso es validar los RNF obtenidos en etapas tempranas del desarrollo de software, haciendo uso de la gestión del conocimiento. En cuanto al proceso de validación, éste se compone de ocho actividades que se relacionan con los roles y productos de trabajo, a través de los cuales se consideran los criterios y las técnicas de validación que pretenden aportar a una adecuada aceptación y acuerdo de los RNF capturados y analizados durante el proceso de desarrollo (Figura 2). Es importante aclarar que el proceso está diseñado para ser utilizado en cualquier empresa desarrolladora de software independiente del tamaño de la misma, así como, aplicado bajo cualquier metodología de desarrollo que se utilice en el proyecto, ya sea tradicional o ágil. 
Figura 2. Proceso de validación de requisitos no funcionales

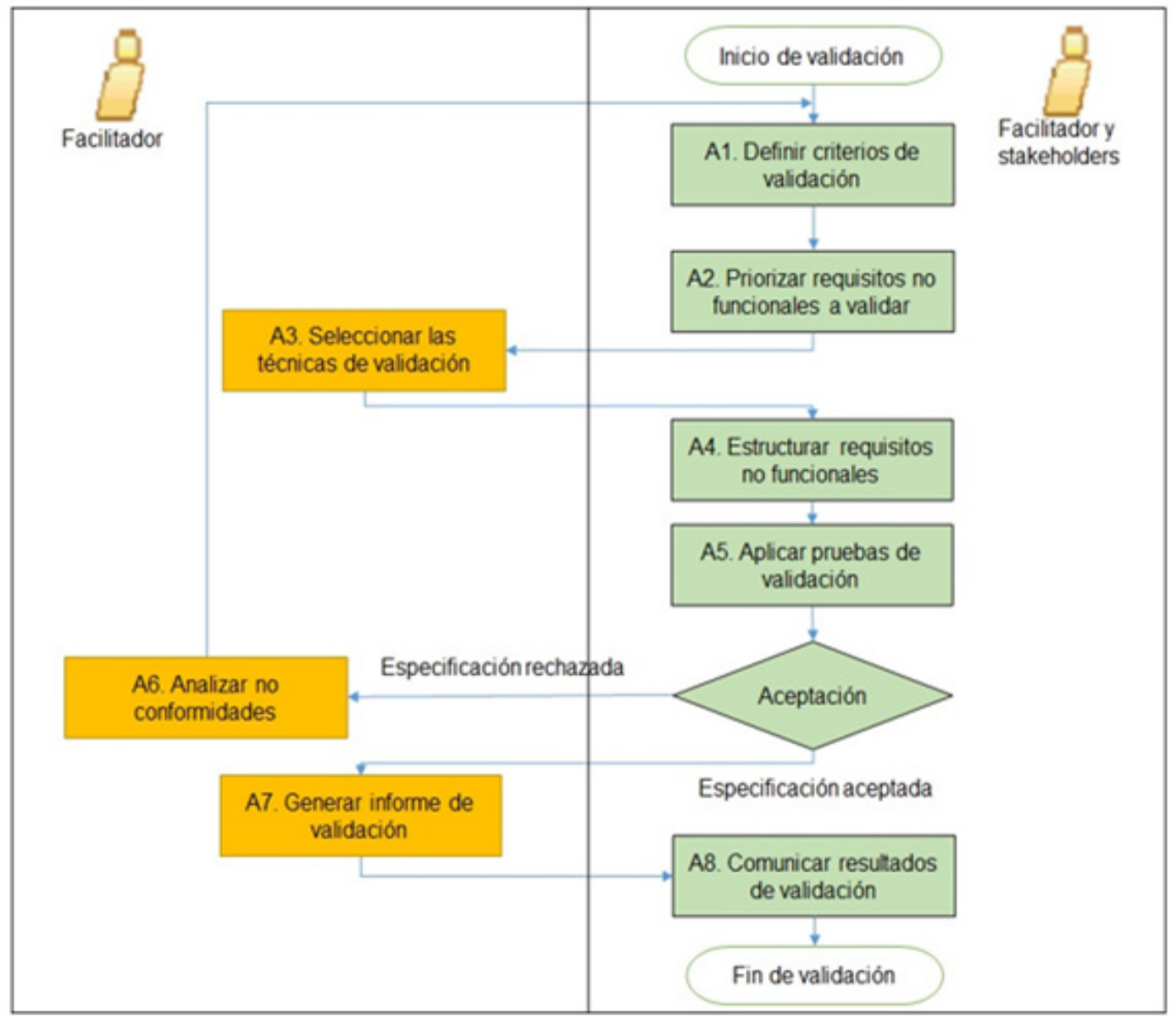

Fuente: Elaboración propia

En este proceso de validación de RNF, el rol facilitador normalmente será un ingeniero de sistemas, el cual podrá también guiar y comunicarse permanentemente con los stakeholders (técnicos, de negocio y de dirección) durante la ejecución de las actividades del proceso. Finalmente, frente a los elementos de gestión de conocimiento, se utilizan los conceptos de ciclo de gestión de conocimiento, flujos de conocimiento y tipos de conocimiento.

Desde el concepto de ciclo de gestión de conocimiento [35], la validación de los RNF implica conocimientos que requieren capturarse y/o crearse, para luego ser compartidos y usados por otras personas para que puedan nuevamente ser tomados en su día a día integrándolos a los conceptos previos con los que contaban. Con respecto a los flujos de conocimiento, se integra el modelo de trasformación de conocimiento SECI [36] el cual expone 4 flujos de conocimiento en una organización: socialización, exteriorización, combinación e interiorización. Estos flujos han permitido describir, para cada actividad del proceso de validación de RNF, cómo cambia el conocimiento frente a los RNF durante la validación de los mismos. Por último, los tipos de conocimiento, aportan al proceso de validación identificando las necesidades de conocimiento que se deben considerar en las diferentes actividades. El proceso de validación de RNF ha sido descrito mediante una estructura llamada marco para la descripción de las actividades, propuesto por [37] el cual permite especificar los aspectos de gestión de conocimiento indicados previamente. 
A continuación, se describen de manera general las actividades del proceso de validación de RNF (Tablas de la 5 a la 12), y se presenta en detalle solo la actividad 4- Estructurar requisitos no funcionales, dado que es la principal actividad del proceso desde la perspectiva de la gestión de conocimiento, esto es, que requiere un mayor grado de implicación de conocimientos previos (tópicos, procedurales y episódicos) cubiertos en las actividades anteriores del proceso para poder ser ejecutada de manera adecuada. (Tabla 8.a, 8.b y 8.c).

Tabla 5. Descripción de la actividad A1 - Definir criterios de validación

\begin{tabular}{|l|l|l|}
\hline \multicolumn{3}{|c|}{ Marco para la descripción las actividades del proceso de Validación de RNF } \\
\hline $\begin{array}{l}\text { Nombre del conocimiento } \\
\text { principal requerido }\end{array}$ & Criterios de validación de RNF \\
\hline Tema de conocimiento & Definir criterios de validación para ser aplicados en el proceso \\
\hline Descripción & $\begin{array}{l}\text { En esta actividad el facilitador socializa a los stakeholders de dirección los criterios que guiarán el } \\
\text { proceso de validación de los requisitos no funcionales y que serán tenidos en cuenta por el grupo } \\
\text { de interesados que participarán en el proceso, de manera que puedan evaluar uniformemente cada } \\
\text { característica de calidad. Es importante conocer e incluir los criterios de validación propios de la } \\
\text { organización que hagan parte de sus políticas y/o lineamientos internos. }\end{array}$ \\
\hline Entradas & $\begin{array}{l}\text { Políticas, procedimientos, formatos } \\
\text { documentos propios de la } \\
\text { organización. }\end{array}$ & \multicolumn{1}{|c|}{ Salidas } \\
\hline
\end{tabular}

Fuente: Adaptado de [37]

Tabla 6. Descripción de la actividad A2 - Priorizar Requisitos No Funcionales a validar

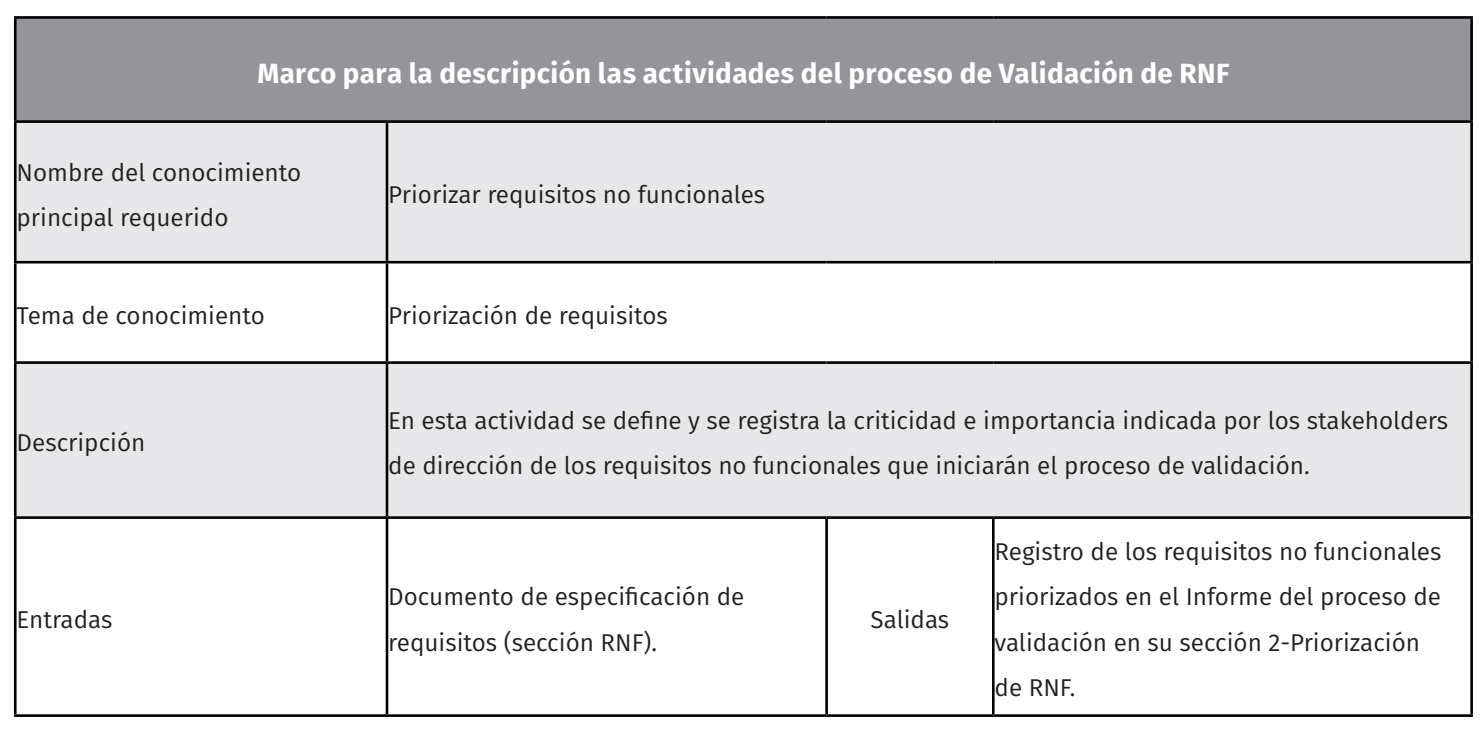

Fuente: Adaptado de [37] 
Tabla 7. Descripción de la actividad A3 - Seleccionar las técnicas de validación

\begin{tabular}{|l|l|l|l|}
\hline \multicolumn{2}{|l|}{ Marco para la descripción las actividades del proceso de Validación de RNF } \\
\hline $\begin{array}{l}\text { Nombre del } \\
\text { conocimiento principal } \\
\text { requerido }\end{array}$ & \begin{tabular}{l} 
Técnicas de validación \\
\hline Tema de conocimiento
\end{tabular} & \begin{tabular}{l} 
Seleccionar las técnicas de validación para RNF \\
\hline Descripción
\end{tabular} & $\begin{array}{l}\text { En esta actividad, el facilitador del proceso de validación identifica los stakeholders técnicos, de negocio y } \\
\text { su nivel de conocimiento acerca de RNF, así como la cultura de la organización de manera que le permita } \\
\text { elegir la(s) técnica(s) de validación más adecuada(s) para la representación de los RNF y su posterior } \\
\text { validación por este grupo de stakeholders. }\end{array}$ \\
\hline Entradas & $\begin{array}{l}\text { Proceso de Validación de RNF sección - } \\
\text { Técnicas de validación }\end{array}$ & \multicolumn{1}{|c|}{ Salidas } & Técnicas seleccionadas. \\
\hline
\end{tabular}

Fuente: Adaptado de [37]

Tabla 8.a. Descripción de la actividad A4 - Estructurar requisitos no funcionales (Adaptado de [37])

\begin{tabular}{|c|c|c|}
\hline \multicolumn{3}{|c|}{ Marco para la descripción la actividad 4- Estructurar RNF } \\
\hline $\begin{array}{l}\text { Nombre del conocimiento principal } \\
\text { requerido }\end{array}$ & \multicolumn{2}{|c|}{ Técnicas de validación de RNF } \\
\hline Tema de conocimiento & \multicolumn{2}{|c|}{ Estructurar los RNF utilizando técnicas de validación } \\
\hline Descripción & \multicolumn{2}{|c|}{$\begin{array}{l}\text { En esta actividad, el facilitador del proceso de validación revisa y analiza el contenido } \\
\text { de los RNF en el documento de especificación de requisitos con el fin de determinar y } \\
\text { estructurar según la(s) técnica(s) de validación seleccionada(s) en la actividad anterior } \\
\text { (A3) el ¿qué? y el ¿cómo? mostrar la información relevante y pertinente a cada tipo de } \\
\text { stakeholders y nivel de conocimiento frente a los RNF. Además, el facilitador toma el } \\
\text { conjunto de criterios de aceptación previamente definidos en la actividad A1 y genera las } \\
\text { pruebas de validación para tales RNF. }\end{array}$} \\
\hline \multicolumn{2}{|l|}{ Entradas } & Salidas \\
\hline \multicolumn{2}{|c|}{$\begin{array}{l}\text {-Documento de especificación de requisitos (sección } \\
\text { requisitos No Funcionales) }\end{array}$} & $\begin{array}{l}\text {-Registro de los RNF de manera estructurada a través de las plantillas A y } \\
\text { B (Figura 3). }\end{array}$ \\
\hline \multicolumn{2}{|l|}{ - Técnicas de validación definidas } & -Definición de los Casos de prueba para los RNF. \\
\hline \multicolumn{2}{|l|}{-Criterios de validación } & \\
\hline \multicolumn{2}{|l|}{-Plantilla A- Escenario de negocio } & \\
\hline \multicolumn{2}{|l|}{-Plantilla B- Atributos de Calidad. } & \\
\hline \multicolumn{2}{|c|}{-Plantilla de casos de prueba de la organización } & \\
\hline
\end{tabular}

Fuente: Adaptado de [37] 
Tabla 8.b. Descripción de la actividad A4 - Estructurar requisitos no funcionales

\begin{tabular}{|c|c|c|c|c|c|}
\hline \multicolumn{6}{|c|}{ Marco para la descripción la actividad 4- Estructurar RNF } \\
\hline \multicolumn{6}{|c|}{ Conocimiento involucrado en: } \\
\hline \multirow{5}{*}{ Actividad } & \multirow{5}{*}{$\begin{array}{l}\text { A4. Estructurar } \\
\text { requisitos no } \\
\text { funcionales }\end{array}$} & \multirow{5}{*}{$\begin{array}{l}\text { Tipo de } \\
\text { conocimiento }\end{array}$} & Creado & & \\
\hline & & & Utilizado & $\mathrm{x}$ & \\
\hline & & & Generado & & \\
\hline & & & Incrementado & & \\
\hline & & & Requerido & $x$ & \\
\hline \multirow{4}{*}{ Rol(es) } & \multirow{3}{*}{$\begin{array}{l}\text { - Facilitador } \\
\text { del proceso de } \\
\text { validación de } \\
\text { RNF }\end{array}$} & \multirow{4}{*}{$\begin{array}{l}\text { Nivel de } \\
\text { conocimiento }\end{array}$} & Principiante & & $\mathrm{x}$ \\
\hline & & & Competente & & \\
\hline & & & Experto & $x$ & \\
\hline & - Stakeholders & & Maestro & & \\
\hline \multicolumn{6}{|c|}{ Descomposición del tipo de conocimiento } \\
\hline \multirow{2}{*}{ Tópico } & \multicolumn{5}{|c|}{$\begin{array}{l}\text { - Técnicas de validación de RNF: procedimiento que permite a los usuarios detectar y resolver conflictos entre } \\
\text { requisitos no funcionales, verificando cada uno de los criterios de validación. }\end{array}$} \\
\hline & \multicolumn{5}{|c|}{$\begin{array}{l}\text { - Requisitos no funcionales: definen las propiedades ambientales y las restricciones de implementación relacionadas } \\
\text { con el desempeño del producto software. }\end{array}$} \\
\hline \multirow{5}{*}{ Procedural } & \multicolumn{5}{|c|}{ Esta actividad comprende las siguientes tareas: } \\
\hline & \multicolumn{5}{|c|}{$\begin{array}{l}\text { - A4T1: Revisar la información de los RNF descritos en el documento de especificación para seleccionar la información } \\
\text { relevante a cada tipo de stakeholders. }\end{array}$} \\
\hline & \multicolumn{5}{|c|}{$\begin{array}{l}\text { - A4T2: Estructurar la información de los RNF obtenida dependiendo de la técnica de validación escogida y utilizando } \\
\text { las plantillas A y B del proceso. }\end{array}$} \\
\hline & \multicolumn{5}{|c|}{$\begin{array}{l}\text {-A4T3: Preparar pruebas de validación considerando los criterios de validación definidos apoyándose de la plantilla } \\
\text { para el diseño de los casos de prueba. }\end{array}$} \\
\hline & \multicolumn{5}{|c|}{ - A4T4: Socializar los RNF estructurados y los casos de prueba definidos a los stakeholders. } \\
\hline
\end{tabular}

Fuente: Adaptado de [37] 
Tabla 8.c. Descripción de la actividad A4 - Estructurar requisitos no funcionales (Adaptado de [37])

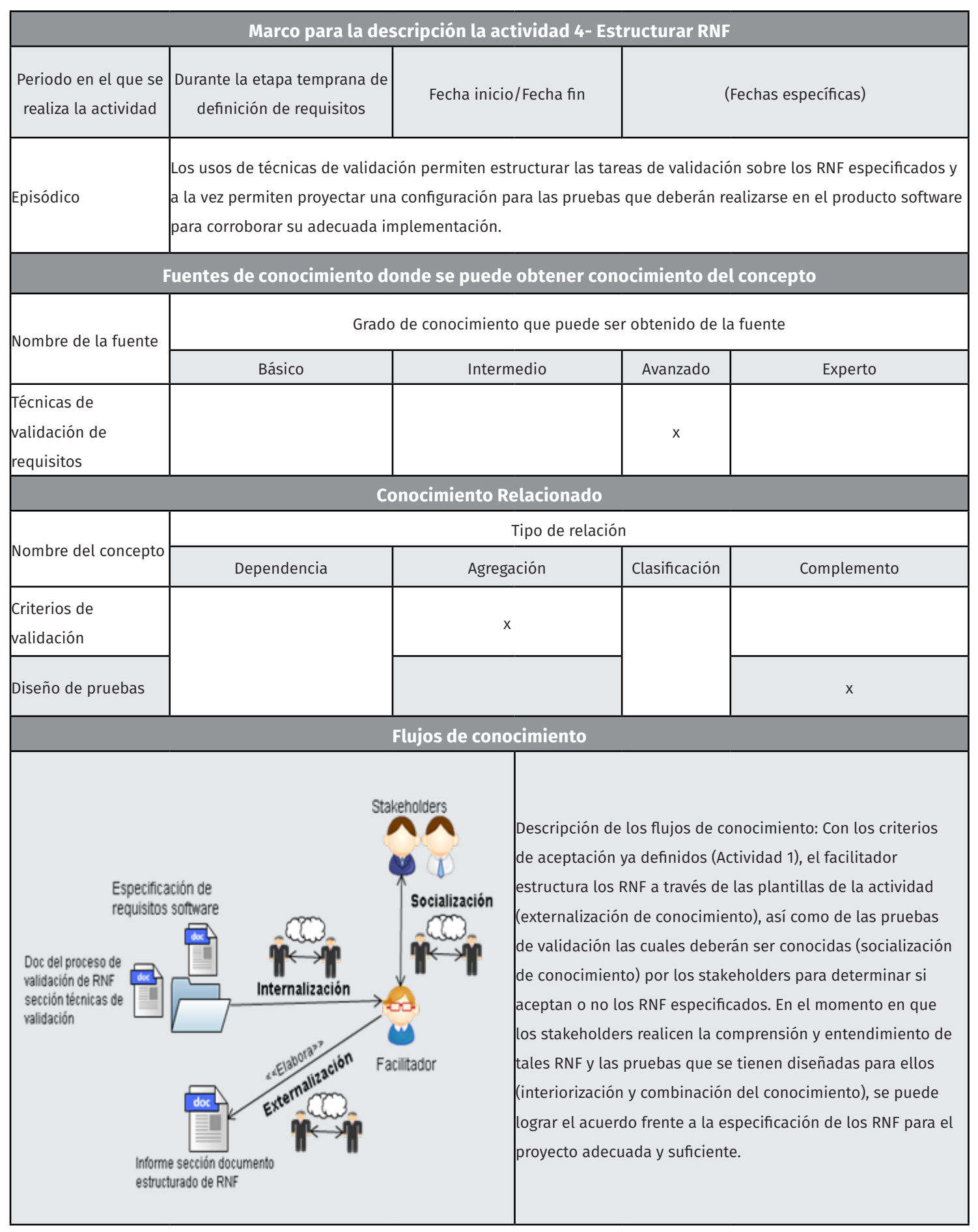

\section{Fuente: Adaptado de [37]}


Tabla 9. Descripción de la actividad A5 - Aplicar pruebas de validación (Adaptado de [37])

\begin{tabular}{|c|c|c|c|}
\hline \multicolumn{4}{|c|}{ Marco para la descripción las actividades del proceso de Validación de RNF } \\
\hline $\begin{array}{l}\text { Nombre del conocimiento } \\
\text { principal requerido }\end{array}$ & \multicolumn{3}{|l|}{ Pruebas de validación de RNF } \\
\hline Tema de conocimiento & \multicolumn{3}{|c|}{ Aplicar pruebas de validación de RNF especificados } \\
\hline Descripción & \multicolumn{3}{|c|}{$\begin{array}{l}\text { En esta actividad, se ejecutan y evalúan las pruebas de validación para determinar la aceptación c } \\
\text { no del documento de especificación de RNF. }\end{array}$} \\
\hline \multirow[b]{2}{*}{ Entradas } & $\begin{array}{l}\text { - Informe del proceso de validación } \\
\text { sección 4-Estructuración de RNF. }\end{array}$ & \multirow[b]{2}{*}{ Salidas } & - Plantillas pruebas de validación diligenciadas \\
\hline & - Plantillas pruebas de validación & & $\begin{array}{l}\text { - Registro de los resultados de pruebas de } \\
\text { validación en el Informe del proceso en su } \\
\text { sección 5-Reporte Pruebas de validación de } \\
\text { RNF (Informe del stakeholders de dirección). }\end{array}$ \\
\hline
\end{tabular}

Fuente: Adaptado de [37]

Tabla 10. Descripción de la actividad A6 - Analizar no conformidades

\begin{tabular}{|c|c|c|c|}
\hline \multicolumn{4}{|c|}{ Marco para la descripción las actividades del proceso de Validación de RNF } \\
\hline $\begin{array}{l}\text { Nombre del conocimiento principal } \\
\text { requerido }\end{array}$ & \multicolumn{3}{|l|}{ Pruebas de validación de RNF } \\
\hline Tema de conocimiento & \multicolumn{3}{|c|}{ Analizar no conformidades de las pruebas de validación. } \\
\hline Descripción & \multicolumn{3}{|c|}{$\begin{array}{l}\text { En esta actividad el facilitador analiza el informe del proceso de validación para identificar } \\
\text { las inconsistencias y así poderlas comunicar al organismo correspondiente, con el objetivo de } \\
\text { que revise, refine, mejore y/o corrija la especificación de los RNF para dar solución a las no } \\
\text { conformidades presentadas en el proceso de validación, y además el facilitador levanta las } \\
\text { lecciones aprendidas del desarrollo de todo el proceso de validación con el fin de mejorar el } \\
\text { proceso para la siguiente ejecución. }\end{array}$} \\
\hline Entradas & $\begin{array}{l}\text { Informe del proceso de validación } \\
\text { sección 5-Pruebas de validación de } \\
\text { RNF. }\end{array}$ & Salidas & $\begin{array}{l}\text { Registro de gestión de cambios del } \\
\text { proceso de validación de requisitos no } \\
\text { funcionales en el Informe del proceso } \\
\text { de validación, en su sección } 6 \text {-Gestión } \\
\text { de cambios del proceso de validación } \\
\text { de RNF. }\end{array}$ \\
\hline
\end{tabular}

Fuente: Adaptado de [37] 
Tabla 11. Descripción de la actividad A7 - Generar informe de validación

\begin{tabular}{|c|c|c|c|}
\hline \multicolumn{4}{|c|}{ Marco para la descripción las actividades del proceso de Validación de RNF } \\
\hline $\begin{array}{l}\text { Nombre del conocimiento principal } \\
\text { requerido }\end{array}$ & \multicolumn{3}{|l|}{ Gestión de reportes } \\
\hline Tema de conocimiento & \multicolumn{3}{|c|}{ Generar informe del proceso de validación de RNF } \\
\hline Descripción & \multicolumn{3}{|c|}{$\begin{array}{l}\text { En esta actividad el facilitador consolida todos los documentos generados durante el } \\
\text { proceso de validación de RNF. }\end{array}$} \\
\hline Entradas & $\begin{array}{l}\text { Documentos generados en la ejecución } \\
\text { del proceso. }\end{array}$ & Salidas & $\begin{array}{l}\text { Reporte completo del proceso de } \\
\text { validación de RNF. }\end{array}$ \\
\hline
\end{tabular}

Fuente: Adaptado de [37]

Tabla 12. Descripción de la actividad A8 - Comunicar resultados de validación

\begin{tabular}{|l|l|l|l|}
\hline \multicolumn{3}{|c|}{ Marco para la descripción las actividades del proceso de Validación de RNF } \\
\hline Nombre del conocimiento principal requerido & Proceso de validación de RNF \\
\hline Tema de conocimiento & \begin{tabular}{l} 
Comunicación de resultados de validación de RNF \\
\hline Descripción
\end{tabular} & $\begin{array}{l}\text { En esta actividad el facilitador comunica el desarrollo y resultados del proceso de } \\
\text { validación de RNF. }\end{array}$ \\
\hline Entradas & $\begin{array}{l}\text { Informe proceso } \\
\text { de validación de } \\
\text { RNF. }\end{array}$ & Salidas & $\begin{array}{l}\text { Acta de aceptación del documento de } \\
\text { especificación de RNF. }\end{array}$ \\
\hline
\end{tabular}

Fuente: Adaptado de [37]

Figura 3- Plantillas A y B (salidas de la actividad A4: Estructurar requisitos no funcionales)

\begin{tabular}{|c|c|c|c|}
\hline \multicolumn{4}{|c|}{ Escenario de Negocio } \\
\hline & \multicolumn{3}{|c|}{$\begin{array}{l}\text { Identificador del Escenario: } \\
\text { Identificador Único del Escenario de negocio. } \\
\text { Ejemplo. ESC-01 }\end{array}$} \\
\hline & \multicolumn{3}{|c|}{$\begin{array}{l}\text { Descripción del Escenario: } \\
\text { Descripolion del escenario de negocio. }\end{array}$} \\
\hline & REFF Relacionados: & $\begin{array}{l}\text { Identificadon(es) de o } \\
\text { negocio. } \\
\text { Elempla: RNF-001 }\end{array}$ & los RNF que pertenecen al escenario de \\
\hline & \multicolumn{2}{|c|}{$\begin{array}{l}\text { Facilitador: } \\
\text { Rol encargaso de realizar y dirigir of } \\
\text { processo de validación de RNF. }\end{array}$} & $\begin{array}{l}\text { Historico de cambios: } \\
\text { Fechas en las que se realzan ajustes al } \\
\text { Escenario de negocio (dd/mm/aaas) }\end{array}$ \\
\hline & \multicolumn{3}{|c|}{ Resipuesta al escenario } \\
\hline & \multicolumn{2}{|c|}{ Simulación } & Respuesta \\
\hline & \multicolumn{2}{|c|}{$\begin{array}{l}\text { Oripen del estimulo: } \\
\text { Es ia entidad (un usuaria, un sistema. } \\
\text { otc.) que produce eil estimula. }\end{array}$} & \multirow[t]{2}{*}{$\begin{array}{l}\text { Respuesta: } \\
\text { Actividad que se leva a cabo luego de } \\
\text { equ el estimulo logue al sistema. }\end{array}$} \\
\hline & \multicolumn{2}{|c|}{$\begin{array}{l}\text { Estimulo: } \\
\text { Es el activador (una solohud, una } \\
\text { operacion, un ataque) del escenario. }\end{array}$} & \\
\hline & \multicolumn{2}{|c|}{$\begin{array}{l}\text { Entorno: } \\
\text { Condión en la que so encuerta of } \\
\text { sistema al prosentarve el estimula. }\end{array}$} & \multirow[t]{2}{*}{$\begin{array}{l}\text { Medida: } \\
\text { Merica definida para el too de estimulo y } \\
\text { poder determinar un valor cuantflcable. }\end{array}$} \\
\hline & \multicolumn{2}{|c|}{$\begin{array}{l}\text { Artefacto: } \\
\text { Componente o destinatario que recobe el } \\
\text { estimulo (puede ser lodo sistema, una o } \\
\text { mis partes) }\end{array}$} & \\
\hline
\end{tabular}

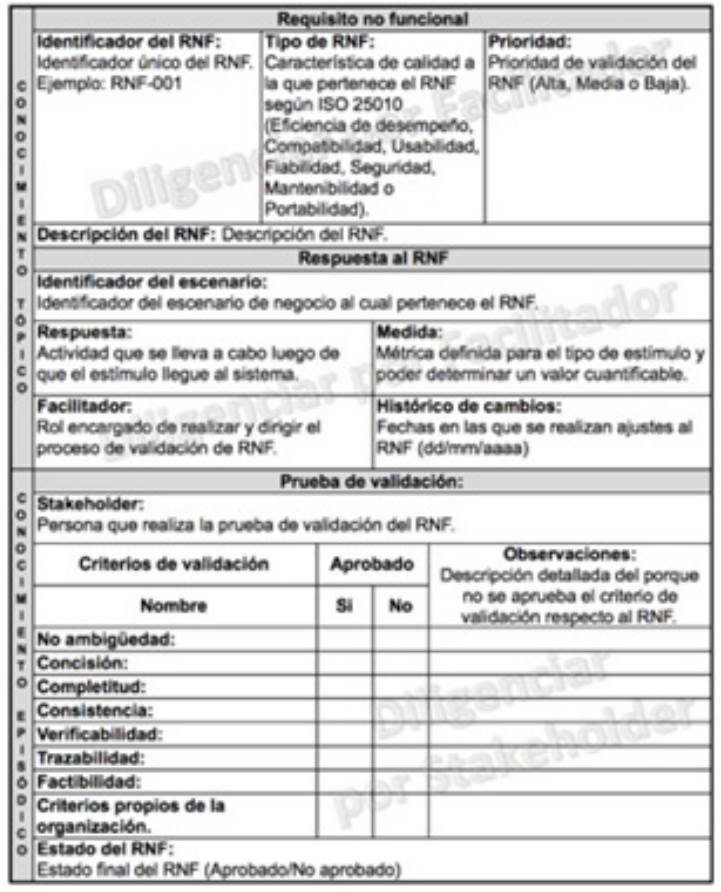

Fuente: Elaboración propia 


\section{Evaluación del proceso a través de Focus group}

Para realizar la evaluación preliminar del proceso sea aplica el método de Focus Group siguiendo la propuesta presentada en [38]. A continuación, se describe de manera sintetizada éste proceso de evaluación, el cual se desarrolló bajo las siguientes actividades: (i) definición del objetivo de evaluación, (ii) preparación de materiales y métodos, (iii) definición de grupos de discusión, (iv) conducción de la sesión de debate, (v) análisis de Información y reporte de resultados. El objetivo de evaluación, es medir la idoneidad del proceso propuesto en términos de claridad, aplicabilidad y relevancia para el desarrollo de software. Para lo cual se elaboró un documento resumen de la propuesta, el cual fue enviado a los participantes del focus de manera previa y de acuerdo a una agenda para la ejecución de la sesión. Así mismo, se elaboró un formato de encuesta con el propósito registrar las medidas cuantitativas y cualitativas de la evaluación. Estas medidas incluyen los criterios de evaluación: claridad, aplicabilidad y relevancia para los proyectos de desarrollo. Estos criterios utilizaron una escala de 1 a 3, donde 1 es No cumple, 2 es cumple moderadamente y 3 es cumple completamente.

A partir de estos materiales y la agenda planeada, se invita a 8 profesionales, de los cuales asisten 5 , quienes deberían contar con alguno de los siguientes dos perfiles: un primer perfil, con experiencia en la industria del software y con conocimientos en el proceso de captura y validación de requisitos, y un segundo perfil con experiencia en el rol de cliente de un proceso de desarrollo de software y que haya participado en proceso de captura y validación de requisitos. Al momento de la conducción de la sesión, se decide grabar en audio y video la totalidad del debate para no perder detalles del mismo, así mismo se toman notas de las apreciaciones de cada uno de los participantes.

\section{Resultados del Focus Group}

A través de los instrumentos definidos para la sesión, se obtienen resultados cualitativos en términos de puntos de mejora y ventajas de la propuesta. Entre los puntos de mejora están: simplificar el proceso, considerar metodologías ágiles para mejorar su aplicabilidad, definir de manera más concreta los roles propuestos, incluir una manera de homogenizar el conocimiento previo de los participantes frente al conceptos técnicos requeridos, ajustar el nombramiento de las actividades 4 y 5 , considerar formas de operación de empresas que no están centralizadas. Como ventajas de la propuesta se obtienen: el proceso de validación de RNF reduciría los posibles sobrecostos que podrían surgir al identificar una mala descripción de un RNF en la etapa de diseño o desarrollo de software, el proceso permite dar valor a los RNF dentro de las empresas, permite llevar a cabo un conjunto de actividades claras, y organizadas para validar los RNF, la propuesta podría incluso apoyar la validación de los requisitos funcionales, permite incluir restricciones y componentes de calidad que implícitamente esperan los stakeholders y, la inclusión de técnicas de validación aporta en aumentar la calidad y eficiencia del proceso de desarrollo de software.

Desde la perspectiva cuantitativa, y teniendo en cuenta la escala definida para los criterios de evaluación definidos (de 1 a 3), se obtuvieron los siguientes resultados por parte de los encuestados, a partir de calcular el promedios de las calificaciones registradas (Tabla 13). 
Tabla 13. Resultados de calificaciones obtenidas a través del formato de evaluación

\begin{tabular}{|l|c|c|c|}
\hline \multirow{2}{*}{\multicolumn{2}{c|}{}} & \multicolumn{3}{c|}{ Criterios de evaluación } \\
\cline { 2 - 4 } & Claridad & Aplicabilidad & Relevancia \\
\hline Participante 1 & 3 & 3 & 3 \\
\hline Participante 2 & 3 & 3 & 3 \\
\hline Participante 3 & 3 & 3 & 3 \\
\hline Participante 4 & 3 & 2 & 3 \\
\hline Participante 5 & 2 & 2 & 3 \\
\hline Promedio & 2.8 & 2.6 & 3 \\
\hline
\end{tabular}

Fuente: Elaboración propia

\section{Discusión}

Con base en los resultados cualitativos, se decide adoptar algunas mejoras sugeridas al proceso de validación inicial, entre las cuales están: cambio de nombre en la actividad A4 por Estructurar requisitos no funcionales, cambio de nombre en la actividad A5 por Aplicar pruebas de validación, se incluye la aclaración de la aplicabilidad del proceso de validación frente al tamaño de las empresas y a las metodologías de desarrollo, y se aclara que el proceso de validación y plantillas diseñadas se podrían utilizar para reemplazar todo el documento de especificación de requisitos y/o complementarlo.

Los resultados cuantitativos permitieron evidenciar que el proceso propuesto podría ser idóneo para los proyectos de desarrollo de software, dada la baja importancia o profundidad que generalmente se les da a éstos requisitos en las fases iniciales del desarrollo, así mismo se pudo determinar que el proceso de validación de RNF es claro y fácil de comprender en su descripción, aunque se deben considerar las sugerencias recibidas frente a simplificarlo, para aumentar su probabilidad de aplicación en pequeñas organizaciones. Desde la perspectiva de la gestión de conocimiento, los expertos confirman que el proceso podría ser de gran ayuda para tener unos RNF correctamente entendidos por parte de los stakeholders, lo cual sugiere una evaluación satisfactoria de las actividades que componen el proceso de validación de RNF; las cuales fueron estructuradas de manera sólida a través de elementos concretos de la disciplina de conocimiento.

\section{Amenazas a la validez y limitaciones}

Para hacer un análisis de la validez de la propuesta, se consideran los aspectos que se describen a continuación:

- Validez de constructo: Para las preguntas utilizadas en el focus group se han definido medidas objetivas que podrían permitir la obtención de información fiable acerca de los criterios de claridad, aplicabilidad y relevancia del proceso de validación de RNF en proyectos de desarrollo de software. Se recolectó además información cualitativa a través de una encuesta y la observación participativa de los diferentes expertos.

- Validez interna: El análisis de la información recolectada consistió en comparar los resultados tanto cualitativos como cuantitativos obtenidos en la sesión, de manera que pudiera determinarse la idoneidad del proceso de validación de RNF. Las conclusiones se construyen a partir de los datos recolectados mediante la sesión de debate y la encuesta establecida del método empírico utilizado. 
- Validez externa: El diseño del Focus Group puede ser replicado en otros proyectos de investigación dado que cuenta con un protocolo que cubre el objetivo de evaluación, unos materiales y métodos definidos, la conformación del grupo de discusión, una agenda para la sesión de debate, y una actividad de análisis de resultados.

Entre las limitaciones a considerar en el Focus Group se tiene que, la cantidad de expertos es baja, lo cual afecta la confiabilidad de los resultados en cierta medida, sin embargo, estos expertos si contaban con alta experiencia en el desarrollo de software, conocimiento adecuado en técnicas de validación y en el concepto de proceso.

\section{Conclusiones}

En este artículo se ha presentado el proceso para la validación de RNF basado en la gestión de conocimiento, como una alternativa para que las organizaciones desarrolladoras de software cuenten con un mecanismo para validar estos requisitos que son generalmente omitidos en las etapas tempranas del desarrollo. El proceso para la validación de RNF, consta de 8 actividades, las cuales consideran diferentes elementos y aspectos concretos de la gestión de conocimiento. Como resultado de la evaluación del proceso de validación de RNF, se puede evidenciar que es un proceso, que basado en la gestión del conocimiento, puede brindar a los Stakeholders una nueva perspectiva de aporte a los procesos de ingeniería de software desde su propia visión frente a los requisitos no funcionales. El proceso ofrece una plantilla marco para la descripción las actividades del proceso de Validación de RNF permitiendo capturar eficientemente la información de todo el proceso de validación, con el fin de poder gestionar y asegurar la generación constante de nuevos conocimientos, lo cual podría seguir mejorando el desarrollo de este proceso. Es un proceso que, como consecuencia de su posible puesta en marcha dentro de las organizaciones, permitiría un mayor grado de explicitud y dominio de los RNF al interior de las mismas de manera que aporta como mecanismo de mejora del desarrollo del software y la calidad del software. Además, es un proceso técnico que puede ser utilizado para reemplazar métodos tradicionales en la etapa de elicitación de requisitos, permitiendo la simplificación de estas actividades al poder establecer un formato único el cual pueda servir para ser gestionado (utilizado) en estas actividades, también, la estructuración de los RNF en los escenarios podría ser el insumo para realizar el diseño de los casos de prueba en la etapa respectiva dentro del ciclo de desarrollo.

Este trabajo de investigación está enmarcado dentro del proyecto MERliNN [39], un framework para la elicitación de requisitos no funcionales basado en la gestión de conocimiento, el cual pretende ser un mecanismo para identificar, capturar y especificar requisitos no funcionales en proyectos de desarrollo de software. Este framework está compuesto por diferentes componentes, entre los cuales están: (i) un componente de representación de RNF, que actualmente aún se encuentra en construcción y que busca facilitar la captura y especificación de estos requisitos a través de signos lingüísticos y, (ii) el proceso para la validación de RNF, presentado en éste artículo. El propósito general del proyecto es ofrecer componentes especializados que permitan la gestión de los RNF en la industria del software, de manera que estos requisitos puedan ser visibles y tener un mayor grado de entendimiento por parte de los diferentes interesados del proyecto, especialmente por los interesados de negocio. En este sentido, el proceso de validación de RNF se integrará al componente de representación de RNF para fortalecer la validación de estos requisitos.

Finalmente, como trabajos futuros se determinarán métricas bajo metodologías de medición que permitan medir cada criterio de validación presentado en la propuesta frente a las necesidades reales de los 
stakeholders, asi como trabajar en alivianar el proceso y posteriormente realizar una segunda evaluación del mismo en un contexto empresarial mediante un estudio de caso.

\section{Referencias bibliográficas}

1. ISO/IEC, “ISO/IEC 25010 Systems and software engineering -- Systems and software Quality Requirements and Evaluation (SQuaRE) -- System and software quality models," First Edition ed, 2011.

2. L. E. Mendoza, M. A. Perez, A. Griman, and M. Ortega, “Análisis del Impacto del Proceso de Desarrollo en las Características de Calidad de Software," in Ideas, 2003.

3. K. Pohl, Requirements engineering: fundamentals, principles, and techniques: Springer Publishing Company, Incorporated, 2010.

4. S. Dragicevic, S. Celar, and L. Novak, "Use of method for elicitation, documentation, and validation of software user requirements (MEDoV) in agile software development projects," in 2014 Sixth International Conference on Computational Intelligence, Communication Systems and Networks, 2014, pp. 65-70, IEEE.

5. R. A. Zahniser, "Building software in groups" American Programmer, vol. 3(7-8), 1990.

6. K. Wiegers and J. Beatty, Software requirements: Pearson Education, 2013.

7. T. Ambreen, N. Ikram, M. Usman, and M. Niazi, “Empirical research in requirements engineering: trends and opportunities" Requirements Engineering, vol. 23(1), pp. 63-95, 2018/03/01 2018.DOI: 10.1007/ s00766-016-0258-2.

8. F. Ricca, M. Torchiano, M. Di Penta, M. Ceccato, and P. Tonella, "Using acceptance tests as a support for clarifying requirements: A series of experiments" Information and Software Technology, vol. 51(2), pp. 270-283, 2009.

9. D. M. Berry and E. Kamsties, "Ambiguity in requirements specification," in Perspectives on software requirements, ed: Springer, 2004, pp. 7-44.

10. H. U. Khan, I. Asghar, S. A. A. Ghayyur, and M. Raza, "An empirical study of software requirements verification and validation techniques along their mitigation strategies" Asian Journal of Computer and Information Systems, vol. 3(3), 2015.

11. A. Casamayor, D. Godoy, and M. Campo, "Identification of non-functional requirements in textual specifications: A semi-supervised learning approach" Information and Software Technology, vol. 52(4), pp. 436-445, 2010.

12. P. Anitha and B. Prabhu, "Integrating requirements engineering and user experience design in product life cycle management," in 2012 First International Workshop on Usability and Accessibility Focused Requirements Engineering (UsARE), 2012, pp. 12-17, IEEE.

13. T. Dings $\varnothing y r$, F. O. Bjфrnson, and F. Shull, "What do we know about knowledge management? Practical implications for software engineering” IEEE Software, vol. 26(3), pp. 100-103, 2009.

14. A. Aurum, F. Daneshgar, and J. Ward, "Investigating Knowledge Management practices in software development organisations-An Australian experience" Information and Software Technology, vol. 50(6), pp. 511-533, 2008.

15. L. Mathiassen and P. Pourkomeylian, "Managing knowledge in a software organization" Journal of Knowledge Management, vol. 7(2), pp. 63-80, 2003.

Revista Investigación e Innovación en Ingenierías, Vol. 8, n³, pp. 50-71, 2020. DOI: https://doi.org/10.17081/invinno.8.3.4704 
16. O. Rodríguez-Elías and A. Martínez García, "Diseño de sistemas y estrategias de gestión del conocimiento: Un enfoque metodológico orientado a procesos y flujos de conocimiento" Editorial Académica Española, 2011.

17. F. J. Pino, M. Piattini, and G. Horta Travassos, "Managing and developing distributed research projects in software engineering by means of action-research" Revista Facultad de Ingeniería Universidad de Antioquia, (68), pp. 61-74, 2013.

18. K. Petersen, R. Feldt, S. Mujtaba, and M. Mattsson, "Systematic Mapping Studies in Software Engineering," in EASE, 2008, pp. 68-77.

19. V. Gervasi and B. Nuseibeh, "Lightweight validation of natural language requirements" Software: Practice and Experience, vol. 32(2), pp. 113-133, 2002.

20. T. H. Nguyen, B. Q. Vo, M. Lumpe, and J. Grundy, "KBRE: a framework for knowledge-based requirements engineering" Software Quality Journal, vol. 22(1), pp. 87-119, 2014.

21. M. M. Marciszack, R. Pérez, and C. Castro, "Validación de Requerimientos a través de Modelos Conceptuales," in XV Workshop de Investigadores en Ciencias de la Computación, 2013.

22. M. Ahmad, N. Belloir, and J.-M. Bruel, "Modeling and verification of functional and non-functional requirements of ambient self-adaptive systems" Journal of Systems and Software, vol. 107pp. 50-70, 2015.

23. J. Zhou, Y. Lu, and K. Lundqvist, "The observer-based technique for requirements validation in embedded real-time systems," in 2014 IEEE 1st International Workshop on Requirements Engineering and Testing (RET), 2014, pp. 47-54, IEEE.

24. J. Domínguez, P. R. Dapena, and S. SoftWcare, "Verificación de los requisitos no funcionales en el software crítico," in Forum Calidad, 2003, pp. 25-31.

25. M. A. Chaves, "La ingeniería de requerimientos y su importancia en el desarrollo de proyectos de software" InterSedes: Revista de las Sedes Regionales, vol. 6(10), pp. 1-13, 2005.

26. O. Romero and A. Abelló, "Automatic validation of requirements to support multidimensional design" Data \& Knowledge Engineering, vol. 69(9), pp. 917-942, 2010.

27. E. Parra, C. Dimou, J. Llorens, V. Moreno, and A. Fraga, "A methodology for the classification of quality of requirements using machine learning techniques" Information and Software Technology, vol. 67pp. 180-195, 2015.

28. A. Kokune, M. Mizuno, K. Kadoya, and S. Yamamoto, "FBCM: Strategy modeling method for the validation of software requirements" Journal of Systems and Software, vol. 80(3), pp. 314-327, 2007.

29. E. Bjarnason, P. Runeson, M. Borg, M. Unterkalmsteiner, E. Engström, B. Regnell, et al., "Challenges and practices in aligning requirements with verification and validation: a case study of six companies" Empirical software engineering, vol. 19(6), pp. 1809-1855, 2014.

30. I. Sommerville, "Ingeniería de software" 2011.

31. IEEE, "IEEE 830 Recommended Practice for Software Requirements Specifications," ed. USA and Canada: IEEE Computer Society, 1998.

32. ISO/IEC/IEEE, "ISO/IEC/IEEE 29148:2011 Systems and software engineering -- Life cycle processes -Requirements engineering," vol. 2018, Fist Edition ed, 2011.

33. R. R. Young, The requirements engineering handbook: Artech House, 2004. 
34. ISO/IEC, "ISO/IEC 25023:2016 Systems and software engineering - Systems and software Quality Requirements and Evaluation (SQuaRE) - Measurement of system and software product quality," ed, 2016, p. 45.

35. K. Dalkir, Knowledge management in theory and practice: Routledge, 2013.

36. I. Nonaka, R. Toyama, and N. Konno, “SECI, Ba and leadership: a unified model of dynamic knowledge creation" Long range planning, vol. 33(1), pp. 5-34, 2000.

37. B. Flores-Ríos, "Model de evolución de la gestión del conocimiento en mipymes, de acuerdo al nivel de madurez en un programa de mejora de procesos de software" Universidad Autónoma de Baja California, 2016.

38. M. Mendoza-Moreno, C. González-Serrano, and F. J. Pino, “Focus group como proceso en ingeniería de software: una experiencia desde la práctica" Dyna, vol. 80(181), pp. 51-60, 2013.

39. S. L. Buitrón, B. L. Flores-Rios, and F. J. Pino, "Elicitación de requisitos no funcionales basada en la gestión de conocimiento de los stakeholders" Ingeniare. Revista chilena de ingeniería, vol. 26(1), pp. 142-156, 2018. 